\title{
Bilateral Traumatic Globe Luxation with Optic Nerve Transection
}

\author{
Levent Tok $^{\mathrm{a}}$ Ozlem Yalcin Tok ${ }^{\mathrm{a}}$ Tugba Cakmak Argun ${ }^{\mathrm{a}}$ Omer Yilmaz \\ Alime Gunes $^{a} \quad$ Elif Nisa Unlu ${ }^{b}$ Sezgin Sezer ${ }^{c}$ Seda Ibisoglu \\ Mehmet Argun ${ }^{a}$ \\ Departments of ${ }^{a}$ Ophthalmology and ${ }^{b}$ Radiology, Faculty of Medicine, Süleyman Demirel \\ University, Isparta, and 'Department of Ophthalmology, Burdur State Hospital, \\ Burdur, Turkey
}

\section{Key Words}

Optic nerve transection - Optic nerve avulsion · Traumatic globe luxation · Orbital trauma

\begin{abstract}
Purpose: The purpose of this study was to document clinical findings and management of a patient with bilateral globe luxation and optic nerve transection. Materials and Methods: A 25-year-old female patient was admitted to the emergency department with bilateral traumatic globe luxation following a motor vehicle accident. Results: Visual acuity testing showed no light perception. The right pupil was dilated and bilaterally did not react to light. The globes were bilaterally intact. A computed tomography scan revealed Le Fort type II fractures, bilateral optic nerve transection and disruption of all extraocular muscles. The globes of the patient were bilaterally reduced into the orbit. However, the patient developed phthisis bulbi in the right eye at month 3. Conclusion: Globe luxation presents a dramatic clinical picture, and may lead to the development of severe complications due to the concomitance of complete optic nerve dissection and multiple traumas. Even if the luxated globe is repositioned into the orbit, there is still an increased risk of the development of phthisis due to ischemia.

(c) 2014 S. Karger AG, Basel
\end{abstract}

\section{Introduction}

Globe luxation is a rare condition. It may result from a finger poke into the orbit during sports injuries or severe orbital and facial fractures from motor vehicle accidents $[1,2]$. Auto-enucleation or attempted traumatic enucleation by gouging injuries has been reported in assaults, including those performed by individuals with psychotic disorders or drug-abuse 
Tok et al.: Bilateral Traumatic Globe Luxation with Optic Nerve Transection

problems [3]. Periorbital soft tissue laxity, shallow orbits, exophthalmos and eyelid manipulation are risk factors that contribute to globe luxation. Spontaneous luxation also has been reported in pathological conditions such as craniofacial dysostosis, Grave's disease, orbital tumors and floppy eye lid [2, 3].

In the study presented here, we report a case of luxation of the globe out of its socket with bilateral optic nerve avulsion transection resulting from a motor vehicle accident.

\section{Case Report}

A 25-year-old female patient was admitted to the emergency service with multiple traumas following a motor vehicle accident. The patient was reported to have been riding in the back seat of the vehicle without the safety belt fastened when the vehicle rolled down an embankment. The patient was conscious but agitated. Bilateral globes were completely out of their sockets (fig. 1). During examination, the right pupil was dilated and bilaterally did not react to light. The patient had no light perception bilaterally. The right globe was intact in appearance but hypotonic. The anterior segment of the left globe was normal; however, the right fundus could not be visualized. The left eye fundus examination revealed patches of hemorrhage around the optic disk. The patient had deep cuts of $4 \mathrm{~cm}$ towards the right lateral section of the upper lip and $5 \mathrm{~cm}$ on the lower jaw.

A cranial and orbital tomography revealed multiple comminuted fractures of the nasal bones, ethmoidal bone, both zygomatic bones, hard palate, right maxillary alveolar bone, and right pterygoid process. The fracture arch passed through the whole walls of both orbits except for the orbital roofs, all walls of the right maxillary sinus and all walls of the left maxillary sinus except for the anterior wall were classified as Le Fort II fracture. There were also fractures of the bony septum and the sphenoid sinus. All paranasal sinuses were filled with blood. There was a displaced fracture of the right mandibular ramus and angulation of the condyle to the medial aspect. There was a comminuted fracture of the left mandibular fossa resulting from impaction of the condyle.

The patient had an intraorbital hematoma in the right eye. There was rupture of all extraocular muscles of both eyes, except for the superior and lateral rectus of the left eye. Bilateral optic nerves were avulsed at the orbital apex and retracted toward the posterior bulbus (fig. 2). The right optic nerve was retracted $2.5 \mathrm{~cm}$ to the nasal side and the left optic nerve was retracted $2 \mathrm{~cm}$. Brain parenchyma was normal.

In addition, the left femoral head was dislocated. The patient underwent closed reduction and derotational casting at the orthopedic clinic. She was instructed by the plastic surgery unit to do jaw-opening and closing exercises for mandibular damage.

Approximately $10 \mathrm{~h}$ after hospital admission, the patient underwent reduction of the globe into the orbit under general anesthesia. In the meantime, the globes were preserved and kept wet using artificial tear gel. Rather than a major surgery, it was planned to perform the reduction of the globe into the orbit because of the existence of bilateral transection in the optic nerves of the patient, visual hopelessness, posterior extraocular muscle rupture and the risk of not finding the ruptured muscles. During the operation, the socket was enlarged with lateral canthotomy, and then the globes were gently repositioned into the orbit with the assistance of an orbital plate and Desmarres lid retractor. The patient underwent bilateral temporary tarsorrhaphy, and a compression pad and bandage were applied. The compression closure was removed on day 3 and the tarsorrhaphy sutures on day 10. There was progressive and unresponsive edema in the right cornea. On the subsequent days, an ulcer developed, accompanied by the shrinkage of the globe on the 
Tok et al.: Bilateral Traumatic Globe Luxation with Optic Nerve Transection

lower half of the cornea (fig. 3). Phthisis bulbi occurred in the right eye at month 3. A prosthesis was fit over the phthisis bulbi (fig. 4). An anterior segment examination of the left eye was unremarkable except for pupillary dilation. There was an upward and outward deviation of the left eye. Her eye movements were limited in all directions of gaze. Fundus examination revealed optic atrophy.

\section{Discussion}

In globe luxation, the extraocular muscles and optic nerve can be affected at different levels. The extraocular muscles and optic nerve can remain intact, as in patients with spontaneous globe luxation; or they can be damaged at different levels of severity, as in most patients with traumatic globe luxation. The presence of optic nerve avulsion worsens the prognosis. There can be partial or complete avulsion of the optic nerve. In cases of complete optic nerve avulsion, the optic sheath, which is more elastic than the optic nerve, usually remains attached to the globe and the optic nerve may appear normal [4]. Thus, the clinician should consider this in a patient with vision loss. Even though the lamina cribrosa is anatomically the weakest part, optic nerve transection 30-50 $\mathrm{mm}$ behind the globe has been reported in most well documented cases of globe luxation [4, 5]. In the case presented here, there was a transection $25 \mathrm{~mm}$ behind the globe in the right eye, and $20 \mathrm{~mm}$ behind the globe in the left eye, and the optic nerve was found to have been retracted behind the globe. In globe luxation, extraocular muscle loss accompanies almost all cases of nerve damage. The most commonly avulsed extraocular muscles are, in descending order of frequency, medial, inferior, superior, lateral rectus and oblique. The muscles that are most easily found inside the Tenon's capsule, and can be sutured back to their original insertions, are superior, inferior and lateral rectus. In this case, all extraocular muscles of both eyes were avulsed, except for the superior and lateral rectus of the left eye.

A number of mechanisms have been proposed to explain the occurrence of globe luxation. Morris et al. [1] reported that an elongated or wedge-shaped object entering the orbit medially caused globe luxation, accompanied by elongation or rupture of the optic nerve by using or not using the nasal wall as a fulcrum. Multiple fractures of facial and orbital bones can lead to compression in the posterior portion of the orbit, reduced orbital volume and displacement of the eyeball out of its socket [2].

Due to the association of globe luxation with severe craniofacial trauma or posterior optic nerve transection, there is often comorbidity with life-threatening complications such as subarachnoid hemorrhage, meningitis, cerebrospinal fluid leak or basal ganglia infarction $[2,3]$. Therefore, a detailed orbital and cranial scan is important to evaluate the extent of the damage and determine the need for subsequent interventions.

The preservation of visual function is the primary goal in cases of globe luxation. The clinician should take quick action in cases of incomplete avulsion of the optic nerve. In addition, the orbicularis oculi muscle, which may exert pressure on the optic nerve and vascular tissues, should be relaxed $[2,6]$. However, due to the absence of visual function, cosmetic concerns become important in cases of complete optic nerve avulsion. In these patients with no visual function, a variety of approaches have been proposed to discuss whether the eyeball should be preserved. If the globe is intact, the most widely approved strategy is to reposition the globe and suture the muscles back to their normal anatomical position $[2,4-6]$. There are also cases reported in the literature where this has been done for primary enucleation $[1,7]$. 


\section{Case Reports in Ophthalmology}

\begin{tabular}{l|l}
\hline Case Rep Ophthalmol 2014;5:429-434 \\
\hline DOI: 10.1159/000370043 & $\begin{array}{l}\text { @ 2014 S. Karger AG, Basel } \\
\text { www.karger.com/cop }\end{array}$ \\
\hline
\end{tabular}

Tok et al:: Bilateral Traumatic Globe Luxation with Optic Nerve Transection

Globe reduction may not always be sufficient to maintain the future viability of the globe. In such cases, the simultaneous association of transection of the optic nerve and disruption of extraocular muscles impairs the vascular supply of the globe, which may cause the development of anterior segment ischemia, ocular necrosis or infection during the follow-up period $[6,8]$. The final outcome may be phthisis bulbi as it was in the case presented here.

\section{Disclosure Statement}

None of the authors has any conflicts of interest with the submission. No financial support was received for this submission.

\section{References}

1 Morris WR, Osborn FD, Fleming JC: Traumatic evulsion of the globe. Ophthal Plast Reconstr Surg 2002;18:261-267.

2 Bajaj MS, Kedar S, Sethi A, Gupta V: Traumatic globe luxation with optic nerve transection. Orbit 2000;19:165-170.

3 Hindman HB, Srikumaran D, Halfpenny C, Hirschbein MJ: Traumatic globe luxation and enucleation caused by a human bite injury. Ophthal Plast Reconstr Surg 2007;23:422-423.

4 Pillai S, Mahmood MA, Limaye SR: Complete evulsion of the globe and optic nerve. Br J Ophthalmol 1987;71:69-72.

-5 Kiratli H, Tümer B, Bilgiç S: Management of traumatic luxation of the globe. A case report. Acta Ophthalmol Scand 1999;77:340-342.

6 Bajaj MS, Pushker N, Nainiwal SK, Balasubramanya R: Traumatic luxation of the globe with optic nerve avulsion. Clin Experiment Ophthalmol 2003;31:362-363.

7 Unal S, Argin A, Arslan E, Demirkan F, Aksoy A: Bilateral complete avulsion of ocular globes in a Le Fort III maxillofacial fracture: a case report and review of the literature. Eur J Ophthalmol 2005;15:123-125.

8 Hsu HC, Lai JP: Surgical management of traumatic luxation of the ruptured eyeball. Injury 2004;35:10551058.

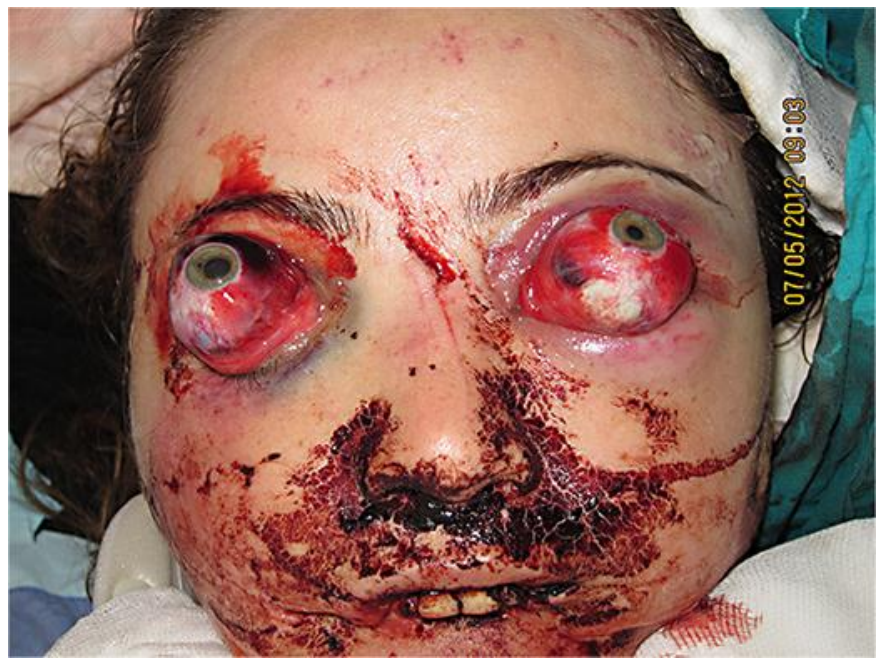

Fig. 1. Patient's appearance on admission. Bilateral globes are outside the orbit with deep facial cuts and deformity of the jaw. 
Tok et al.: Bilateral Traumatic Globe Luxation with Optic Nerve Transection

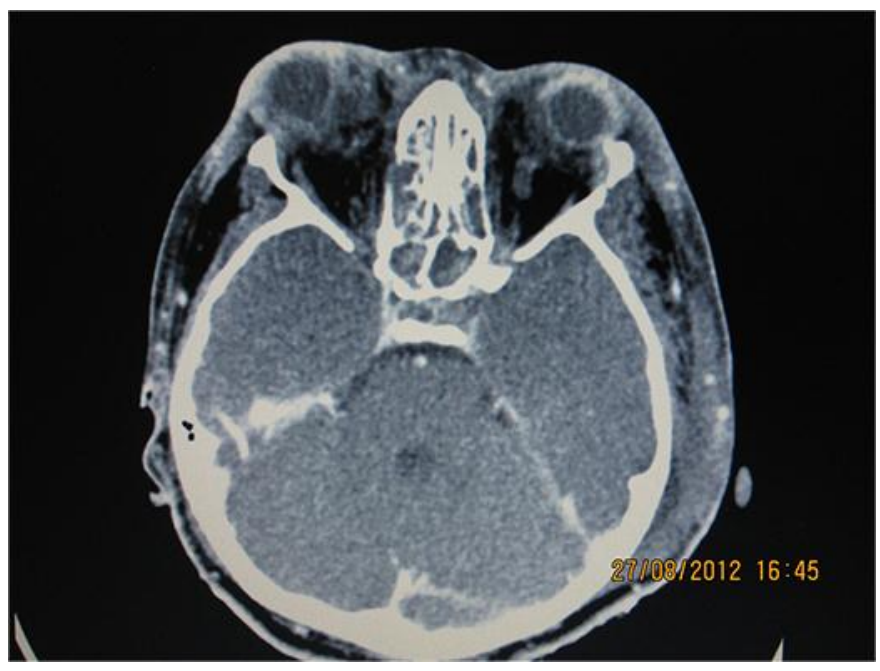

Fig. 2. Axial orbital and cranial computed tomography scan showing bilateral globe luxation, retrobulbar hemorrhage, bilateral optic nerve transection, retraction of the optic nerves behind the globe, avulsion of the lateral and medial rectus and multiple bone fractures.

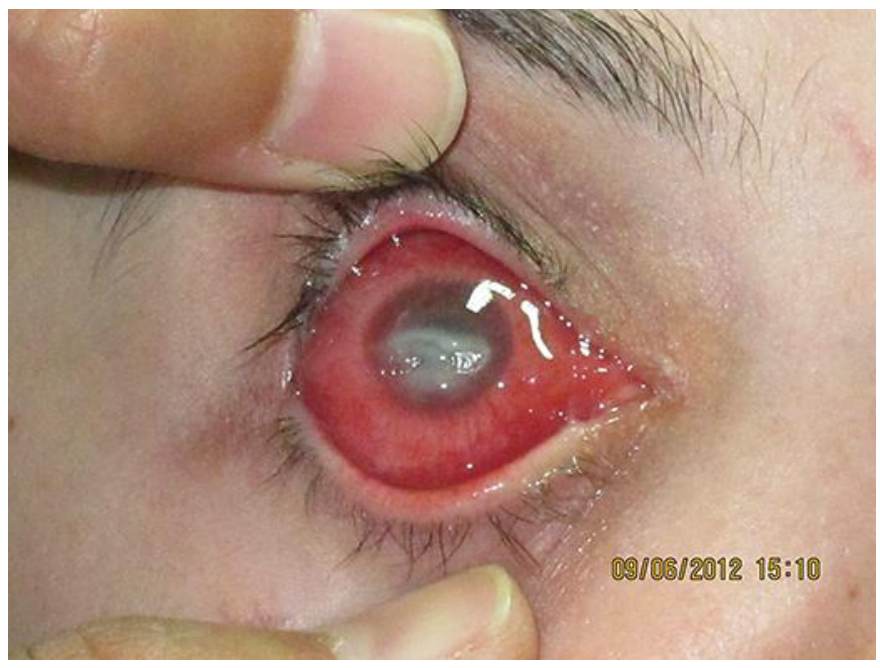

Fig. 3. Anterior segment ischemia and phthisis bulbi in the right eye. 
Case Reports in

Ophthalmology
Case Rep Ophthalmol 2014;5:429-434

DOI: $10.1159 / 000370043$

al: Bilateral Traumatic Globe Lux

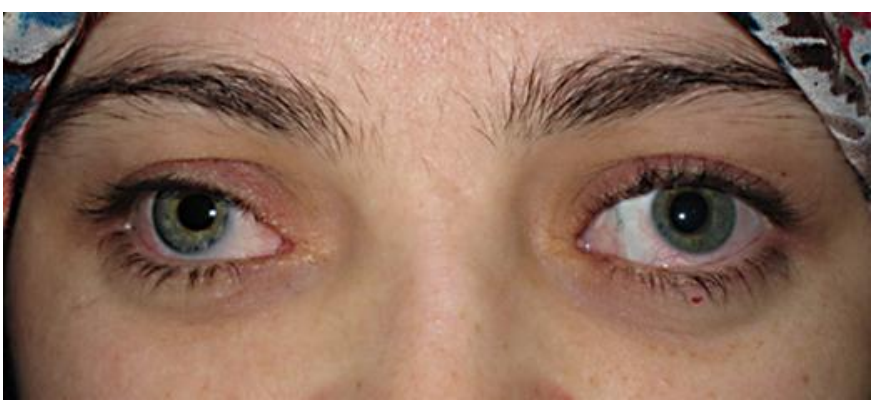

Fig. 4. Patient's appearance after 1 year. She is wearing a prosthesis in the right eye with outward deviation of the left globe. 